Recibido 05 de abril 2021 - Aceptado 01 de Mayo, 2021

\title{
Desgaste profesional y cumplimiento de objetivos de los trabajadores de una institución pública peruana
}

\section{Professional burnout and achievement of objectives of the workers of a Peruvian public institution}

\author{
Alda Mariaca, Edwin Estrada ${ }^{2}$
}

\section{RESUMEN}

El objetivo de la presente investigación fue determinar la relación que existe entre el desgaste profesional y el cumplimiento de objetivos de los trabajadores de la Unidad de Gestión Educativa Local de Tambopata, 2020. La investigación fue cuantitativa, el diseño fue no experimental, correlacional y transversal. La muestra fue conformada por 38 trabajadores de la UGEL Tambopata a quienes se les aplicaron el Cuestionario de Desgaste Profesional y el Cuestionario sobre el Cumplimiento de Objetivos, instrumentos con la validez y confiabilidad requerida. Los hallazgos indican que existe una relación inversa y significativa entre el desgaste profesional y el cumplimiento de objetivos ( $r h o=-0,462 ; p=0,004<0,05$ ). Se concluyó que los altos niveles de desgaste profesional están relacionados con el limitado cumplimiento de objetivos y viceversa.

Palabras clave: Desgaste profesional, cansancio emocional, despersonalización, insatisfacción al logro, cumplimiento de objetivos.

\section{ABSTRACT}

The objective of this research was to determine the relationship between professional burnout and the achievement of objectives of the workers of the Local Educational Management Unit of Tambopata, 2020. The research was quantitative, the design was non-experimental, correlational and transversal. The sample was made up of 38 workers from UGEL Tambopata to whom the Professional Burnout Questionnaire and the Questionnaire on the Compliance of Objectives were applied, instruments with the required validity and reliability. The findings indicate that there is an inverse and significant relationship between professional burnout and the achievement of objectives $(r h o=-0.462 ; p=0.004<0.05)$. It was concluded that the high levels of professional burnout are related to the limited fulfillment of objectives and vice versa.

Keywords: Professional burnout, emotional tiredness, depersonalization, dissatisfaction with achievement, achievement of objectives. 


\section{INTRODUCCIÓN}

El estudio del desgaste profesional tuvo como punto de partida las investigaciones realizadas por Freudenberger (1974) y Maslach (1982), quienes descubrieron que este fenómeno afectaba especialmente a los profesionales (médicos, profesores, enfermeras, psicólogos, etc.) que prestaban servicios y estaban en contacto con otras personas.

Fue definido como un trastorno adaptativo crónico, el cual tiende a hacerse presente cuando las personas están expuestas de manera continua a diversos estresores, ya sean emocionales e interpersonales, exigiendo una alta demanda emocional hacia las personas a las que se les brinda algún tipo de servicio, tal como se mencionó con antelación (Fernández et al., 2017).

Entre las principales secuelas del desgaste profesional están "alteraciones físicas, psicológicas y conductuales relacionadas con el estrés, las cuales derivan normalmente en problemas en el entorno laboral como la hostilidad, dificultad para concentrarse, aumento de las relaciones conflictivas con los compañeros, retraso y ausentismo laboral" (Gilla et al., 2019, p.180).

Maslach y Jackson (1986) determinaron que el desgaste profesional está conformado por tres componentes: el cansancio emocional, la despersonalización y la insatisfacción al logro. El cansancio emocional es definido como el desgaste sostenido de las energías o recursos emocionales que provocan en las personas fatiga, ya sea mental o física. Respecto a la despersonalización, se refiere al desarrollo de sentimientos y actitudes negativas e indiferentes, principalmente hacia los usuarios y otras personas que interactúan con los trabajadores de la UGEL Tambopata. Finalmente, la insatisfacción al logro está asociada a las respuestas negativas que los propios trabajadores tienen sobre sí mismos y hacia la labor que realizan, lo cual puede afectar la relación que tienen con los sus colegas y usuarios.
Respecto al cumplimiento de objetivos, puede ser definido como la concreción de las de acciones que fueron planificadas a inicios de año y que se consideran en el plan estratégico institucional (Arce, 2010). En el caso de la UGEL Tambopata, tiene la finalidad de atender las necesidades educativas en las instituciones educativas pertenecientes a su jurisdicción y es responsabilidad de todos y cada uno de los trabajadores de dicha institución.

Entonces, un objetivo es el punto final al que se debe llegar y es parte importante de una estructura organizacional, de un programa administrativo o de un plan estratégico. Es necesario plantear objetivos, tanto generales como específicos, siendo estos últimos los que especifican la naturaleza propia del primero, por ello se habla de jerarquías (Andía, 2016). Actualmente, toda institución es considerada un sistema en la que participan varios componentes que deben actuar de manera articulada y comprometida para alcanzar los objetivos estratégicos. En ese entender, si las metas u objetivos no están interconectadas con los trabajadores, podría crear caos y desorden en la dinámica de trabajo, y que al final los objetivos pueden terminar distorsionando lo que en verdad se quiere alcanzar.

Así pues, los objetivos deben ser razonables y posibles de ser alcanzados, tienen que estar vinculados a los fines, principios y valores de la institución. Su formulación es necesaria, incluso, se puede aseverar que son para avanzar; son importantes porque son esenciales para su existencia y crecimiento.

Las dimensiones de la variable cumplimiento de objetivos son: planificación, ejecución y evaluación. La planificación de los objetivos es el proceso de establecer objetivos, desarrollar estrategias, trazar los planes de implementación y asignar recursos para alcanzar esos objetivos. En cuanto a la ejecución de los objetivos, se define como el proceso en el cual se ejecuta lo establecido en la planificación, partiendo de los resultados obtenidos en la investigación, para alcanzar los objeti- 
vos propuestos. Finalmente, la evaluación de los objetivos permite verificar si los objetivos planteados previamente se están concretizando. Implica también la aplicación de estándares que son los referentes para medir los objetivos. (Kaplan y Norton, 2006).

El presente estudio permitirá será de mucha utilidad para los directivos de la UGEL Tambopata pues podrán conocer los niveles de desgaste profesional que presentan sus trabajadores, el nivel del cumplimiento de objetivos en la institución y cómo se relacionan ambas variables. En ese sentido, a partir de los hallazgos, la UGEL Tambopata podrá elaborar actividades preventivas como talleres, charlas y capacitaciones para disminuir los niveles de desgaste profesional y así contar con el personal motivado, comprometido y competente que contribuya con el logro de objetivos institucionales.

En virtud a lo expuesto, se consignó como objetivo general determinar la relación que existe entre el desgaste profesional y el cumplimiento de objetivos de los trabajadores de la UGEL Tambopata, 2020.

\section{MATERIALES Y MÉTODOS}

La investigación tuvo un enfoque cuantitativo, ya que para contrastar las hipótesis se realizó la recolección de datos, lo que permitió analizar estadísticamente para luego realizar su sistematización (Sánchez et al., 2018); el diseño fue no experimental, puesto que las variables no fueron manipuladas de manera intencional, sino se observaron tal como se dio en su entorno (Hernández et al. 2014) y el tipo de investigación fue descriptivo correlacional de corte transversal, ya que se analizaron las propiedades y características de ambas variables, se determinó si se relacionaban y la recolección de datos fue en un solo momento, respectivamente (Bisquerra, 2009).

La población y la muestra estuvo conformada por 38 trabajadores, tanto nombrados como contratados del a UGEL Tambopata, cantidad que fue determinada a través de un muestreo censal. Los instrumentos utilizados para la recolección de datos fueron el Cuestionario de Desgaste Profesional y el Cuestionario sobre el Cumplimiento de Objetivos.

El Cuestionario de Desgaste Profesional fue diseñado por Uribe (2010), presenta 30 preguntas de tipo Likert (nunca, casi nunca, a veces, casi siempre y siempre) y se encuentra estructurado en 3 dimensiones: cansancio emocional (ítems 1-9), despersonalización (ítems 10-18) e insatisfacción al logro (ítems 19-30). Sus propiedades métricas se determinaron a través del proceso de validez (V de Aiken: 0,860) y confiabilidad ( $\alpha$ : 0,879). Respecto al Cuestionario sobre el Cumplimiento de Objetivos, fue elaborado por por Mariaca (2020), está conformado por 21 ítems de tipo Likert (nunca, casi nunca, a veces, casi siempre y siempre) y está estructurado en 3 dimensiones: planificación de los objetivos (ítems 1-7), ejecución de los objetivos (ítems 8-14) y evaluación de los objetivos (ítems 15-21). Sus propiedades métricas también se determinaron mediante el proceso de validez (V de Aiken: 0,820) y confiabilidad (a: 0,834).

La recolección de datos se dio en varios momentos, en primera lugar, se explicó el objetivo del estudio y se solicitó el permiso a las autoridades competentes de la UGEL Tambopata. Posteriormente, se contactó a los trabajadores mediante la aplicación de mensajería Whatsapp con el propósito de indicarles la finalidad de la investigación y enviarles el enlace para que puedan acceder a Google Forms, aplicación donde se estructuraron los instrumentos. Cuando ellos accedieron al formulario mencionado, leyeron con atención las orientaciones y brindaron su consentimiento informado, admitiendo que su participación se daba de manera voluntaria. Seguidamente, desarrollaron ambos instrumentos, que tuvo una duración aproximada 15 minutos.

Para realizar el análisis estadístico se optó por trabajar con Software SPSS ${ }^{\varpi}$ versión 22, donde los resultados descriptivos fueron sistematizados en 
tablas de frecuencia y porcentaje. Por otro lado, la parte inferencial se realizó utilizando la prueba no paramétrica rho de Spearman con la finalidad de conocer si ambas variables y dimensiones se relacionaban de manera significativa.

\section{RESULTADOS}

Según la tabla 1, el 39,5\% de los trabajadores presentaban altos niveles de desgaste profesio- nal, el 28,9\% tenían niveles moderados, el 21,1\% evidenciaban muy altos niveles y el 10,5\% mostraban bajos niveles. Respecto a la variable 2, el $42,1 \%$ de los trabajadores indicaron que los objetivos se cumplían casi siempre, el 39,5\% señalaban que a veces, el 15,8\% mencionaron que siempre y el $2,6 \%$ precisaron que casi nunca se cumplían.

Tabla $\mathrm{N}^{\circ} 1$. Niveles de desgaste emocional y cumplimiento de objetivos

\begin{tabular}{lcclccc}
\hline \multirow{2}{*}{ Nivel } & \multicolumn{2}{c}{ Desgaste emocional } & & \multirow{2}{*}{ Nivel } & \multicolumn{2}{c}{ Cumplimiento de objetivos } \\
\cline { 2 - 3 } & $\mathrm{n}$ & $\%$ & & $\mathrm{n}$ & $\%$ \\
\hline Muy bajo & 0 & 0,0 & Nunca & & 0 & 0,0 \\
Bajo & 4 & 10,5 & Casi nunca & 1 & 2,6 \\
Moderado & 11 & 28,9 & A veces & 15 & 39,5 \\
Alto & 15 & 39,5 & Casi siempre & 16 & 42,1 \\
Muy alto & 8 & 21,1 & Siempre & 6 & 15,8 \\
Total & 38 & 100,0 & Total & 38 & 100,0 \\
\hline
\end{tabular}

Fuente: Base de datos

En la tabla 2 se realizó la prueba de normalidad Shapiro - Wilk. Los datos mostraron un p-valor menor a 0,05 para las variables de estudio, lo cual permitió determinar que la muestra no presentaba una distribución normal y por lo tanto era necesario usar una prueba estadística no paramétrica. En este caso se decidió trabajar con el coeficiente de correlación rho de Spearman debido también a la naturaleza y nivel de medición de las variables y dimensiones.

Table No2. Prueba de normalidad Shapiro - Wilk

\begin{tabular}{lccc}
\hline \multirow{2}{*}{ Variables } & \multicolumn{3}{c}{ Kolmogorov-Smirnov $^{\mathrm{a}}$} \\
\cline { 2 - 4 } & Estadístico & gl & Sig. \\
\hline Desgaste profesional &, 976 & 38 &, 003 \\
Cumplimiento de objetivos &, 967 & 38 &, 021 \\
\hline
\end{tabular}

Fuente: Base de datos

En la tabla 3 se puede observar que el coeficiente de correlación rho de Spearman entre las variables desgaste profesional y cumplimiento de objetivos fue de -0,462 con un p-valor inferior al nivel de significancia $(\mathrm{p}=0,004<0,05)$, por ello se rechazó la hipótesis nula y se aceptó la hipótesis alterna que indica que existe una relación inversa y significativa entre ambas variables. 
Tabla N³. Correlación entre el desgaste emocional y el cumplimiento de objetivos

\begin{tabular}{llc}
\hline & & Cumplimiento de objetivos \\
\hline Desgaste & Coeficiente de correlación &,$- 462^{* *}$ \\
emocional & Sig. (bilateral) &, 004 \\
& $\mathrm{~N}$ & 38 \\
\hline
\end{tabular}

**. La correlación es significativa en el nivel 0,01 (2 colas).

Fuente: Base de datos

En la tabla 4 se observa que existe correlación inversa y significativa entre la variable cumplimiento de objetivos y las dimensiones cansancio emocional $(r h o=-0,366 ; p<0,05)$, despersonalización ( $r h o=$ $-0,405 ; \mathrm{p}<0,05)$ e insatisfacción al logro $(r h o=-0,498 ; \mathrm{p}<0,05)$.

Tabla $N^{\circ} 4$. Correlación entre las dimensiones del desgaste profesional y la variable cumplimiento de objetivos

\begin{tabular}{llc}
\hline & & Cumplimiento de objetivos \\
\hline \multirow{2}{*}{ Cansancio emocional } & Coeficiente de correlación &,$- 366^{*}$ \\
& Sig. (bilateral) &, 024 \\
& $\mathrm{~N}$ & 38 \\
\hline \multirow{2}{*}{ Despersonalización } & Coeficiente de correlación &,$- 405^{*}$ \\
& Sig. (bilateral) &, 012 \\
& $\mathrm{~N}$ & 38 \\
\hline \multirow{3}{*}{ Insatisfacción al logro } & Coeficiente de correlación &,$- 498^{* *}$ \\
& Sig. (bilateral) &, 001 \\
& $\mathrm{~N}$ & 38
\end{tabular}

*. La correlación es significativa en el nivel 0,05 (2 colas).

**. La correlación es significativa en el nivel 0,01 ( 2 colas).

Fuente: Base de datos

\section{DISCUSIÓN}

El objetivo principal de la presente investigación fue determinar la relación que existe entre el desgaste profesional y el cumplimiento de objetivos de los trabajadores de la Unidad de Gestión Educativa Local de Tambopata, 2020.

En primer lugar, se identificó que los trabajadores se caracterizaban por presentar altos niveles de desgaste profesional, es decir, se encontraban cansados emocionalmente, mostraban actitudes de indiferencia frente a las personas que atendían y evidenciaban sentimientos de insatisfacción hacia la labor que desempeñaban.

Lo reportado coincide con los hallazgos de Cotrina y Monteza (2016), quienes encontraron que existía una alta prevalencia de desgaste profesional (burnout) en los profesionales que laboran en enfermería, dentro del módulo de emergencia del Hospital Regional de Lambayeque. Ello sería explicado debido a que ellos desarrollaban sus actividades dentro de un ambiente laboral negativo, emocionalmente tóxico, causado por la interacción permanente con pacientes y sus fa- 
miliares, que por su misma condición tienen una carga negativa que contagia y genera distintos niveles de estrés.

Por otro lado, se identificó que los trabadores se caracterizaban porque casi siempre cumplían con los objetivos, es decir, generalmente cumplían la mayoría de objetivos trazados, sin embargo, existían algunos de ellos que no podía concretizarse por diversos motivos, lo cual afectaría el plan estratégico y operacional de la institución.

Este hallazgo es corroborado por Estrada et al. (2021), quien identificó que los profesionales de la educación mostraban un desempeño satisfactorio y cumplían con los objetivos programados a inicios de año, es decir, realizaban el proceso de planificación de las estrategias, los recursos y materiales, tomando en cuenta las particularidades de cada estudiante como su ritmo y estilo de aprendizaje; asimismo ejecutaban los procesos pedagógicos y didácticos durante las actividades de aprendizaje, participaban activamente en la gestión de la institución educativa y reflexionaban críticamente sobre su desempeño en el aula para su propio crecimiento profesional.

Un hallazgo revelador indica que se determinó que existía una relación inversa y significativa entre el desgaste profesional y el cumplimiento de objetivos de los trabajadores de la Unidad de Gestión educativa Local de Tambopata, 2020. El coeficiente de correlación rho de Spearman entre las variables analizadas fue de $-0,462$ con un $\mathrm{p}$-valor inferior al nivel de significancia $(\mathrm{p}=0,004<0,05)$. Estos datos indican que los altos niveles de desgaste profesional estaban relacionados con el limitado cumplimiento de objetivos y viceversa.

Los resultados expuestos coinciden con lo reportado por diversas investigaciones (Terán, 2017; Barriga, 2015; Mababu, 2016; Serrano et al., 2017; Procel, 2015; Estrada et al., 2021; Choy, 2017; Mogollón y Rojas, 2019) que encontraron que el desgaste profesional incidía negativamente en el desempeño de los trabajadores y por ende, en el cumplimiento de los objetivos que ellos se planteaban así como los objetivos institucionales.

Al respecto, Romero (2019) señala que el desgaste profesional es un tipo de respuesta desfavorable a la presencia del estrés agudo y se evidencia sobre todo en las interacciones, impactando tanto en las personas que brindan algún servicio como quienes lo reciben. Por ello existe una mayor prevalencia en personas que realizan labores de apoyo o servicio social directo, como es el caso de los trabajadores de la Unidad de Gestión educativa Local de Tambopata.

Otro hallazgo importante indica que existe una relación inversa y significativa entre el cansancio emocional y el cumplimiento de objetivos de los trabajadores de la Unidad de Gestión educativa Local de Tambopata, 2020. Así pues, el coeficiente de correlación rho de Spearman entre la dimensión y la variable analizada fue de $-0,366$ con un p-valor inferior al nivel de significancia $(p=0,024<0,05)$. Ello indica que los altos niveles de cansancio emocional estaban relacionados con el limitado cumplimiento de objetivos y viceversa.

El resultado expuesto guarda relación con los hallazgos de algunas investigaciones (Estrada et al., 2021; Barriga, 2015; Mababu, 2016; Rodríguez, 2018) que determinaron que el cansancio emocional incidía negativamente en el desempeño laboral y cumplimiento de objetivos personales e institucionales. Por ello, era necesario tomar las medidas correspondientes que les permitan prevenirla y disminuir su prevalencia.

Del mismo modo, se encontró que existe una relación inversa y significativa entre la despersonalización y el cumplimiento de objetivos de los trabajadores de la Unidad de Gestión educativa Local de Tambopata, 2020. Así pues, el coeficiente de correlación rho de Spearman entre la dimensión y la variable analizada fue de -0,405 con un p-valor inferior al nivel de significancia $(\mathrm{p}=0,012<0,05)$. Por ello, se determinó que altos niveles de despersonalización estaban relaciona- 
dos con el limitado cumplimiento de objetivos y viceversa.

El hallazgo expuesto es coherente con los resultados reportados por diversas investigaciones (Estrada et al., 2020; Barriga, 2015; Rodríguez, 2018) que encontraron que las personas que desarrollaban actitudes negativas e indiferentes hacia los usuarios no concretizaban sus objetivos y no cumplían con las metas propuestas por la institución donde laboraban. De acuerdo a Fuster et al. (2019) los profesionales que presentan altos niveles de desgaste y despersonalización suelen brindar un pésimo servicio, actuar de forma indiferente y no ofrecer un apoyo, es decir, llegan a tener una afectación en su desempeño en el caso de que dicha patología no sea identificada y tratada oportunamente.

Finalmente, se determinó que existe una relación inversa y significativa entre la insatisfacción al logro y el cumplimiento de objetivos de los trabajadores de la Unidad de Gestión educativa Local de Tambopata, 2020. Así pues, el coeficiente de correlación rho de Spearman entre la dimensión y la variable analizada fue de $-0,498$ con un $\mathrm{p}$-valor inferior al nivel de significancia $(\mathrm{p}=0,001<0,05)$. Por ello, se determinó que altos niveles de insatisfacción al logro están relacionados con el limitado cumplimiento de objetivos y viceversa.

Lo expuesto es corroborado por diversos estudios (Estrada et al., 2020; Barriga, 2015; Mababu, 2016; Rodríguez, 2018), los cuales llegaron a concluir que los profesionales que tenían percepciones negativas sobre sí mismos y hacia la labor que realizaban, no llegaban a concretizar sus objetivos y presentaban un desempeño poco adecuado. Al respecto, es necesario mencionar que la insatisfacción al logro, además de afectar el cumplimiento de objetivos, disminuye la motivación de los trabajadores e incrementa el desgano y pesimismo. En suma, el profesional se siente impotente y sin deseos de seguir avanzando, desconfía de sus logros, siente que la profesión o el cargo no le corresponden.

\section{CONCLUSIONES}

1. Se ha determinado que existe una relación inversa y significativa entre las variables desgaste profesional y el cumplimiento de objetivos de los trabajadores de la Unidad de Gestión educativa Local de Tambopata, 2020. El coeficiente de correlación rho de Spearman fue de -0,462 con un $\mathrm{p}$-valor inferior al nivel de significancia $(p=0,004<0,05)$. Lo expuesto implica que cuanto más alto sean los niveles de desgaste profesional de los trabajadores, el cumplimiento de objetivos será más limitado y viceversa.

2. Se ha determinado que existe una relación inversa y significativa entre las dimensiones cansancio emocional, despersonalización e insatisfacción al logro y la variable cumplimiento de objetivos de los trabajadores de la Unidad de Gestión educativa Local de Tambopata, 2020. Los coeficientes de correlación rho de Spearman fueron de $-0,366 ;-0,405$ y $-0,498$ respectivamente con un p-valor inferior al nivel de significancia $(\mathrm{p}<0,05)$.

\section{REFERENCIAS BIBLIOGRÁFICAS}

Andía, W. (2016). Enfoque metodológico para los objetivos estratégicos en la planificación del sector público. Industrial Data, 19(1), 28-32. https:// www.redalyc.org/pdf/816/81650062004.pdf

Arce, L. (2010). Cómo lograr definir objetivos y estrategias empresariales. Perspectivas, 25, 191-201. https://www.redalyc.org/ pdf/4259/425942454010.pdf

Barriga, M. (2015). Relación del síndrome del Burnout y el desempeño laboral de los colaboradores de empresas comercializadoras. (Tesis de pregrado). Pontificia Universidad Católica del Ecuador, Ambato, Ecuador. Recuperado de https://repositorio.pucesa.edu.ec/handle/123456789/1247

Bisquerra, R. (2009). Metodología de la Investigación Educativa. (2a ed.). Madrid: La Muralla S.A. 
Choy, R. (2017). Burnout y desempeño laboral en docentes universitarios de una carrera en una universidad privada de Lima Metropolitana. (Tesis de posgrado). Universidad Peruana Cayetano Heredia, Lima, Perú. Recuperado de https://hdl. handle.net/20.500.12866/1011

Cotrina, L. y Monteza, L. (2016). Estrategias de afrontamiento del Síndrome de Burnout por los profesionales de enfermería del servicio de emergencia del Hospital Regional Lambayeque - Chiclayo 2016. (Tesis de pregrado). Universidad Señor de Sipán, Lambayeque, Perú. Recuperado de https://hdl.handle.net/20.500.12802/3942

Estrada, E., Paredes, Y. y Quispe, R. (2021). El desgaste profesional y su relación con el desempeño de los docentes de educación básica regular. Revista Universidad y Sociedad, 13(4), 361-368. Recuperado de https://rus.ucf.edu.cu/index.php/ rus/article/view/2175

Fernández, J., Peláez, J. \& Peralta, M. (2017). Influencia de factores sociodemográficos, laborales y de estilo de vida sobre los niveles de burnout en personal sanitario de cuidados paliativos. Anales del Sistema Sanitario de Navarra, 40(3), 421-431. http://dx.doi.org/10.23938/assn.0114

Freudenberger, H. (1974). Staff burnout. Journal of Social Issues, 30, 159-166. http://dx.doi.org/10.1111/j.1540-4560.1974.tb00706.x

Fuster, D., Jara, N., Ramírez, E., Maldonado, H., Norabuena, R., y García, A. (2019). Desgaste ocupacional en docentes universitarios mediante el modelo factorial confirmatorio. Propósitos y Representaciones, 7(3), 198-214. https://dx.doi. org/10.20511/pyr2019.v7n3.389

Gilla, M., Giménez, S., Moran, V. y Olaz, F. (2019). Adaptación y validación del Inventario de Burnout de Maslach en profesionales argentinos de la salud mental. LIBERABIT. Revista Peruana de Psicología, 25(2), 179-193. https:// doi.org/https://doi.org/10.24265/liberabit.2019. v25n2.04
Hernández, R. Fernández, C. y Baptista, P. (2014). Metodología de la Investigación. (6 $6^{\mathrm{a}} \mathrm{ed}$.). México: Mc Graw-Hill

Kaplan, V. y Norton, D. (2006). Das Summa Summarum des Management. USA: Harvard Business School Press.

Mababu, R. (2016). The Relationship between Burnout and Job performance in Hospitality Professionals. MEDICA REVIEW. International Medical Humanities Review, 5(1), 17-30. https:// doi.org/10.37467/gka-revmedica.v5.828

Maslach, C. (1982). Burnout: The cost of caring. California: Prentice-Hall Press.

Maslach, C. y Jackson, S. (1986). Maslach Burnout Inventory. California: Consulting Psychologists Press.

Mogollón, J. y Rojas, A. (2019). Síndrome de burnout y desempeño laboral en docentes de I.E nacionales del distrito de Caylloma. (Tesis de pregrado). Universidad Nacional de San Agustín de Arequipa, Arequipa, Perú. Recuperado de http:// repositorio.unsa.edu.pe/handle/UNSA/10591

Procel, M. (2015). Síndrome de Burnout y su influencia en el desempeño laboral de los docentes del Décimo Año de Educación Básica del Colegio Beatriz Cueva de Ayora. (Tesis de pregrado). Universidad Nacional de Loja, Loja, Ecuador. Recuperado de https://dspace.unl.edu.ec/jspui/ handle/123456789/11673

Rodríguez, P. (2018). El síndrome de burnout y el desempeño laboral del personal ejecutivo en BBVA Continental Los Olivos, 2018. (Tesis de pregrado). Universidad San Ignacio de Loyola, Lima, Perú. Recuperado de http://repositorio. usil.edu.pe/handle/USIL/9568

Romero, K. (2019). Empatía y síndrome de desgaste profesional (burnout) en docentes de educación básica regular de Lima Metropolitana. Avances en Psicología, 27(2), 237-254. https:// doi.org/10.33539/avpsicol.2019.v27n2.1801 
Sánchez, H., Reyes, C. y Mejía, K. (2018). Manual de términos en investigación científica, tecnológica y humanística. Lima: Universidad Ricardo Palma. http://repositorio.urp.edu.pe/handle/ $\mathrm{URP} / 1480$

Serrano, M., Rangel, A., Vidal, C., Ureña, Y., Anillo, H. y Angulo, G. (2017). Burnout: Síndrome silencioso que afecta el desempeño laboral de los docentes universitarios. Revista Espacios, 38(55), 28-40. Recuperado de https://www.revistaespacios.com/a17v38n55/17385528.html

Terán, C. (2017). Síndrome de Burnout y rendimiento laboral en enfermeras de la Caja Petrolera de Salud en la ciudad de La Paz. (Tesis de pregrado). Universidad Mayor de San Andrés, La Paz, Bolivia. Recuperado de http://repositorio. umsa.bo/xmlui/handle/123456789/12419

Uribe, J. F. (2010). EDO Escala de Desgaste Ocupacional (Burnout). México: Editorial El Manual Moderno. 